\title{
Go-kit: A TOOL TO ENABLE ENERGY LANDSCAPE EXPLORATION OF PROTEINS
}

\author{
Sridhar Neelamraju,,,,$+ \neq$ David J. Wales, ${ }^{*, \neq}$ and Shachi Gosavi ${ }^{*, \dagger}$ \\ $\dagger$ Simons Centre for the Study of Living Machines, National Centre for Biological Sciences, \\ Tata Institute of Fundamental Research \\ $\ddagger$ University of Cambridge, University Chemical Laboratories, Lensfield Road, Cambridge \\ CB2 1EW, U.K \\ E-mail: sridharn@ncbs.res.in; dw34@cam.ac.uk; shachi@ncbs.res.in
}


Table S.1: List of arguments in gokit.py and conmaps.py modules. Combinations of arguments are important and help generate the right input files.

\begin{tabular}{|c|c|}
\hline Argument & Description \\
\hline \multicolumn{2}{|l|}{ conmaps() } \\
\hline-- cutoff & Cut-off for calculating contact map. \\
\hline --scaling & Scaling for calculating contact map \\
\hline --get_seq & Get sequence from pdb \\
\hline -native & Give CA-CA pdbfile as native file \\
\hline-- aa_pdb & Give all-atom pdbfile \\
\hline --traj & Give trajectory filename. Must end with .xtc or .xyz \\
\hline --traj_list & List of trajectories. Comma separated. No spaces. Must end with .xtc or .xyz. \\
\hline --frames & Give three comma separated integers start,stop, step to skip frames in trajectory. \\
\hline$--x y z \_o u t$ & Give XYZ output file name for extracting trajectory. \\
\hline --get_polar & Get positions of polar residues \\
\hline --get_charged & Get positions of charged residues \\
\hline --get_hp & Get positions of hydrophobic residues \\
\hline-- con_prob & Get contact probabilities from xyz or xtc trajectory. \\
\hline$--p l_{-} f e$ & Plot " Free energy profile". Input file containing only Q values.e.g. .xtc.map. \\
\hline --cluster & rmsd based hierarchical clustering of structures. \\
\hline --get_energies & Get energies from single point OPTIM runs for each frame in xyz trajectory \\
\hline --check_pdb & Give pdb ID. Outputs smog readable pdb \\
\hline-- get_pdb & Give pdb ID. Downloads pdb \\
\hline-- gconmap & Give pdb file. Generate contact map from all atom pdb \\
\hline --pl_map & Plot contact map from gconmap. \\
\hline --renumber_res & Give pdb file. Renumbers residues starting from 1 \\
\hline --ext_pair & Pairs of contacts are supplied externally. Not calculated. \\
\hline \multicolumn{2}{|l|}{ gokit ()} \\
\hline-- CA_rad & Radius for C-alpha atom. Default $=4.0$ \\
\hline- -CAcom & Place C-alpha at COM of backbone \\
\hline --Cb_rad & Statistically derived values by default for attype 2 . \\
\hline$--\mathrm{Kb}$ & Kbond \\
\hline$--\mathrm{Ka}$ & Kangle \\
\hline$--\mathrm{Kd}$ & Kdihedral \\
\hline --cutoff & Cut-off for contact-map generation \\
\hline --scaling & Scaling for mapping to all-atom contact-map. \\
\hline --attype & Number of atom types. E.g. 1 for CA, 2 for CA and CB \\
\hline --btmap & Use Betancourt-Thrumalai parameters for CB-CB interactions. \\
\hline$--\mathrm{CBcom}$ & Put CB at center of mass of side-chain (no hydrogens) \\
\hline --grotop & Gromacs topology file output name. \\
\hline$--a a_{-} p d b$ & all-atom pdbfile e.g. 1qys.pdb \\
\hline --pdbgro & Name for .gro file. \\
\hline$--w$ native & Write native files, CA and CA-CB from all atom PDB file \\
\hline --pl_map & Plot contact map \\
\hline --skip_glycine & Skip putting $\mathrm{C} \beta$ on glycine residues \\
\hline --hphobic & Generate hydrophobic contacts. \\
\hline --interactions & Give file "interactions.dat" for user defined CB-CB interactions \\
\hline --radii & Give file "radii.dat" for user defined $\mathrm{C} \beta$ radii \\
\hline
\end{tabular}


Table S.2: Default values for arguments in Go-kit.

\begin{tabular}{|l|l|}
\hline Argument & Default value \\
\hline \hline conmaps() & \\
\hline --cutoff & $4.5 \AA$ \\
--scaling & 1.2 \\
--CACAsep & 3 \\
--CBCBsep & 2 \\
--CACBsep & 2 \\
--frames & $1,1,1000000$ \\
\hline gokit() & \\
\hline --CA_rad & $4 \AA$ \\
--CAcom & False \\
--Cb_rad & BT statistical radii \\
--Kb & 200 \\
--Ka & 40 \\
--Kd & 1 \\
--cutoff & $4.5 \AA$ \\
--scaling & 1.2 \\
--btmap & False \\
--CBcom & True \\
--grotop & Gromacs topology file output name. \\
--pdbgro & Name for .gro file. \\
--skip_glycine & False \\
--hphobic & False \\
--dsb & False \\
\hline \hline
\end{tabular}




\section{Example input files}

$\begin{array}{cc}\text { interaction.dat } & \text { radii.dat } \\ \text { AA }-0.12 & \text { G } 2.25 \\ \text { AD } 0.18 & \text { A } 2.52 \\ \text { AE } 0.258 & \text { V } 2.93 \\ \text { AG }-0.018 & \text { L } 3.09 \\ \text { AK } 0.12 & \text { I } 3.09 \\ \text { AN } 0.144 & \text { M } 3.09 \\ \text { AP } 0.042 & \text { F } 3.18 \\ \text { AQ } 0.126 & \text { P } 2.78 \\ \text { AR } 0.162 & \text { S } 2.59 \\ \text { AS } 0.09 & \text { R } 2.81 \\ \text { AT } 0 & \text { N } 2.84 \\ \text { CA }-0.156 & \text { Q } 3.01 \\ \text { CC }-0.804 & \text { E } 3.23 \\ \text { CD } 0.228 & \text { W } 3.39 \\ \text { CE } 0.276 & \text { D } 2.79 \\ \text { CF }-0.318 & \text { E } 2.96 \\ \text { CG }-0.054 & \text { H } 3.04 \\ \text { CH }-0.114 & \text { K. } 18 \\ \text { CI }-0.288 & \text { R } 3.28 \\ \text { CK } 0.21 & \text { C } 2.74\end{array}$

\section{Examples of analyses:}

(a) Q vs time plots trajecories:

\$ python conmaps.py --aa_pdb 1qys.pdb --native native_ca.pdb --traj_list 1.xtc,2.xtc

--cutoff 4.5 --scaling 1.2 --ext_pair external_pair --frames 1,100000,1

Files 1.xtc.map, 2.xtc.map. 4.xyz.map, etc. are generated.

(b) Plot histogram:

\$ python conmaps.py --pl_fe 1 .xtc.map

For each trajectory (say 1.xtc), a file with extension ".map" is generated with data contained in Figure S.1

(c) Plot probability contact-maps:

\$ python conmaps.py --con_prob --native native_ca.pdb --ext_pair SMOG.contacts.CG

--traj extract.xyz (OR) --traj traj.xtc --frames 1,1000000,1 


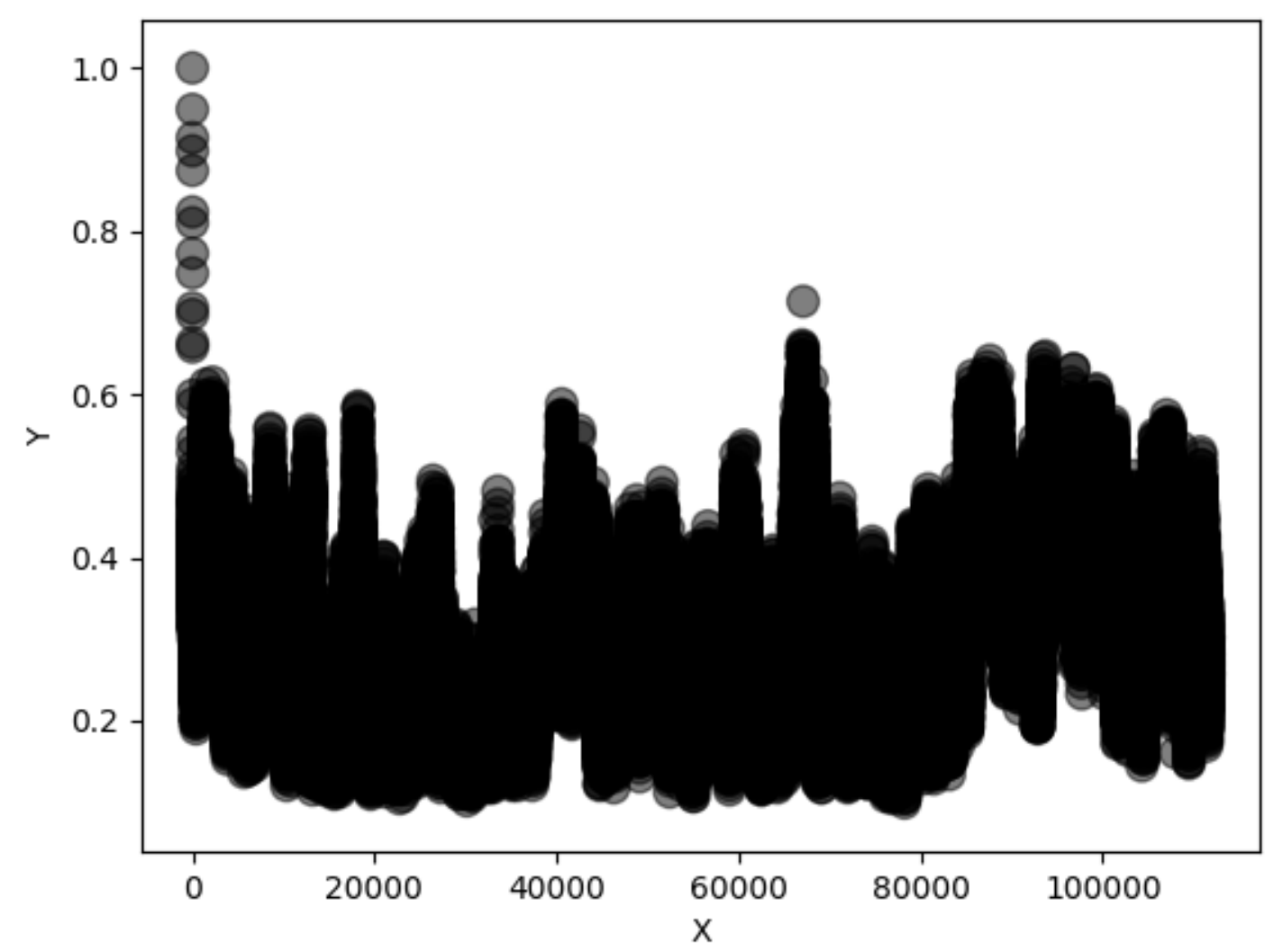

Figure S.1: Evolution of Q vs time plotted from within Go-kit for one trajectory.

\section{GROMACS and PATHSAMPLE details:}

First, we run molecular dynamics simulations in the MD folder generated by Go-kit with the input files generated from Go-kit with GROMACS. Files need to run GROMACS MD are provided in the examples/MDfiles folder in the repository. The SMOG server ${ }^{2}$ contains details on performing MD simulations with GROMACS.

\$grompp -f md.mdp -p gromacs.top -po mdout.mdp -o run.tpr

\$mdrun -x traj.xtc -e ener.edr -o traj.trr -s run.tpr -g mdout.log

-table table_file.xvg -tablep table_file.xvg -tableb table_files/table.xvg > run.out 2> run.err 


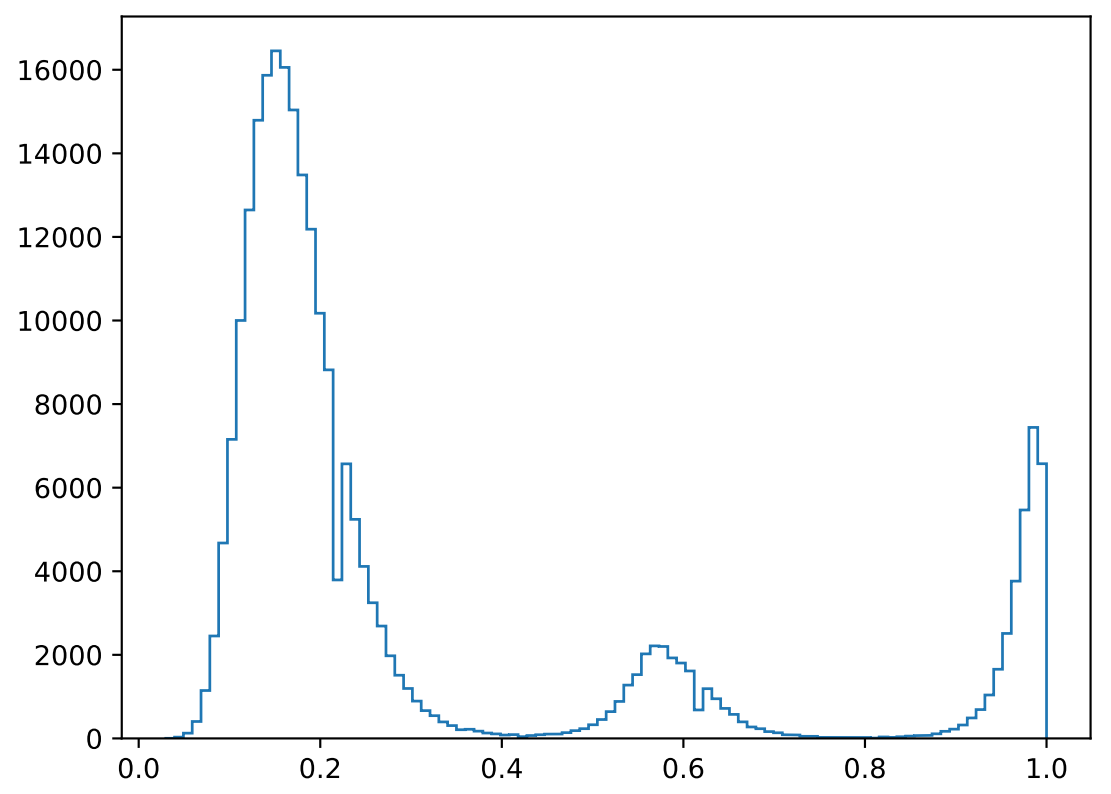

Figure S.2: Histogram generated for .xtc file.

-tableb is required for the desolvation barrier potential. -table points to the table file for the 12-10 LJ potential that is provided with the Go-kit distribution. The trajectory thus generated is clustered into folded, intermediate and transition states with rmsd based clustering plotted with Go-kit. This can also be performed with g_cluster command of GROMACS. \$ python conmaps.py --traj traj.xtc --native native_all.pdb --cluster --frames $1,150000,10$

Next, to run PATHSAMPLE, we extract the a few structures from .xtc file into a .xyz file $\left(\right.$ See MDTraj $\left.{ }^{3}\right)$. These are added to the database by running the command PATHSAMPLE in the PATH folder. For further details, see Ref. ${ }^{4}$ 


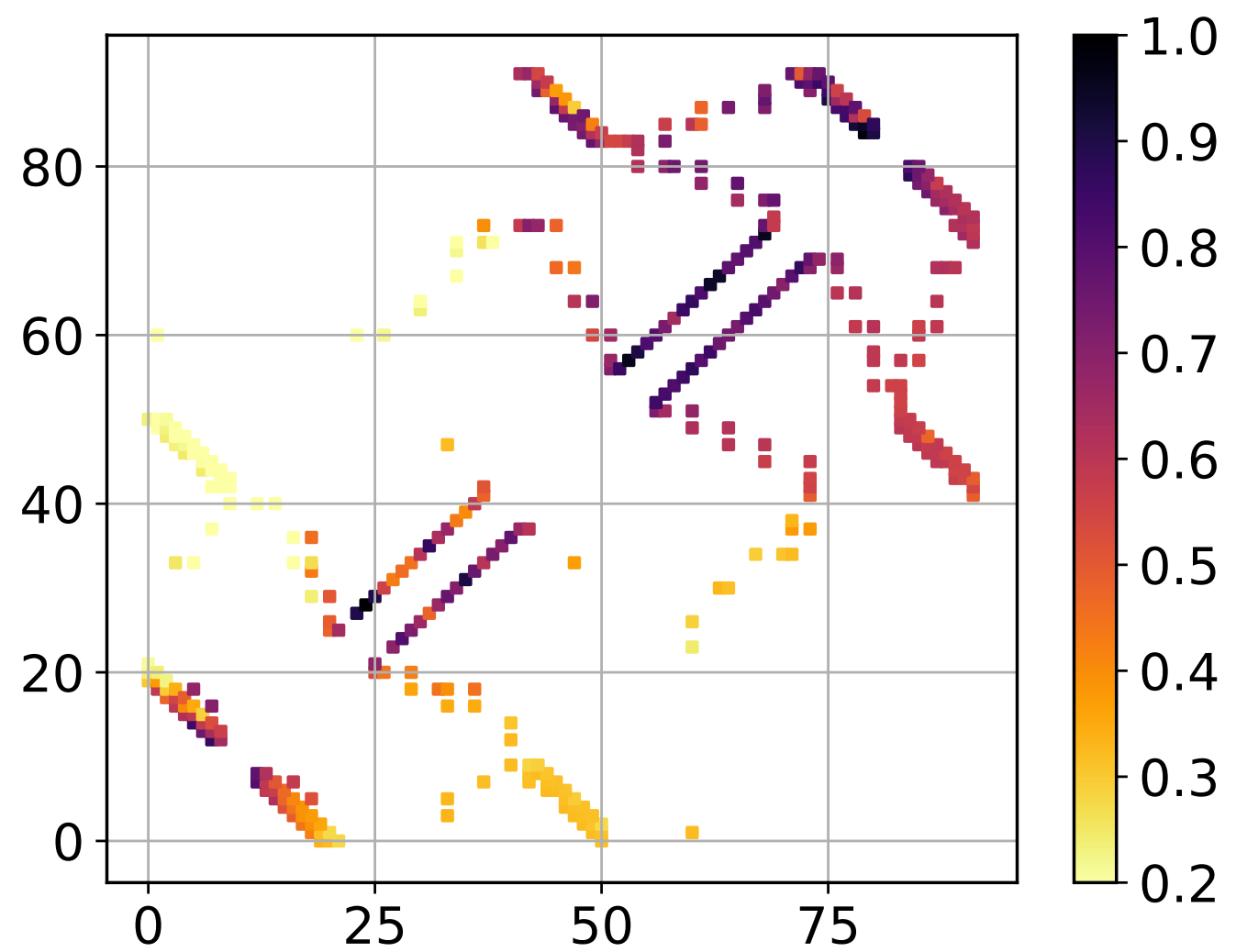

Figure S.3: Probability contact maps can be generated from both MD and DPS trajectories. Example for two MD trajectories of Top7 with two different potentials (top-left:C $\alpha$, bottomright: $\mathrm{C} \alpha+\mathrm{hp})$. Colour indicates probability of formation of the contact. See $^{1}$ for detailed examples.

Table S.3: List of flags and references for potential parameters implemented in Go-kit.

\begin{tabular}{llll}
\hline \hline Model & Reference & Flag & Default potential \\
\hline \hline $\mathrm{C} \alpha$ & 5 & default with attype 1 & $10-12$ \\
$\mathrm{C} \alpha+\mathrm{hp}$ & 6 & - -hphobic & $10-12$ \\
$\mathrm{C} \alpha$ dsb & 7 & - -dsb & desolvation-barrier \\
$\mathrm{C} \alpha$ dsb+hp & 7 & - -dsb --hphobic & desolvation-barrier+gaussian hp \\
$\mathrm{C} \alpha$-C $\beta$ & 8 & default with attype 2 & $10-12$ \\
Betancourt-Thirumalai & 9,10 & - -btmap & $10-12$ \\
Miyazawa-Jernighan & 11 & - -mjmap & $10-12$ \\
\hline
\end{tabular}




\section{References}

(1) Neelamraju, S.; Gosavi, S.; Wales, D. J. Energy Landscape of the Designed Protein Top7. J. Phys. Chem. B 2018, 122, 12282-12291.

(2) Noel, J. K.; Whitford, P. C.; Sanbonmatsu, K. Y.; Onuchic, J. . N. SMOG@ ctbp: Simplified Deployment of Structure-Based Models in GROMACS. Nucleic Acids Res. 2010, 38, W657-W661.

(3) McGibbon, R. T.; Beauchamp, K. A.; Harrigan, M. P.; Klein, C.; Swails, J. M.; Hernández, C. X.; Schwantes, C. R.; Wang, L.-P.; Lane, T. J.; Pande, V. S. MDTraj: A Modern Open Library for the Analysis of Molecular Dynamics Trajectories. Biophysical journal 2015, 109, 1528-1532.

(4) Wales, D. PATHSAMPLE: A Program For Generating Connected Stationary Point Databases and Extracting Global Kinetics. See http://www-wales. ch. cam. ac. uk/PATHSAMPLE 1999, Last accessed: 03rd Mar, 2019.

(5) Go, N. Theoretical Studies of Protein Folding. Annu. Rev. Biophys. Bioeng. 1983, 12, $183-210$.

(6) Chen, T.; Chan, H. S. Native Contact Density and Nonnative Hydrophobic Effects in the Folding of Bacterial Immunity Proteins. PLoS Comput Biol 2015, 11, e1004260.

(7) Liu, Z.; Chan, H. S. Solvation and Desolvation Effects in Protein Folding: Native Flexibility, Kinetic Cooperativity and Enthalpic Barriers Under Isostability Conditions. Phys. Biol. 2005, 2, S75.

(8) Cheung, M. S.; Finke, J. M.; Callahan, B.; Onuchic, J. N. Exploring the Interplay between Topology and Secondary Structural Formation in the Protein Folding Problem. J. Phys. Chem. B. 2003, 107, 11193-11200. 
(9) Reddy, G.; Liu, Z.; Thirumalai, D. Denaturant-Dependent Folding of GFP. Proc. Nat. Acad. Sci. 2012, 109, 17832-17838.

(10) Betancourt, M. R.; Onuchic, J. N. Kinetics of Proteinlike Models: The Energy Landscape Factors that Determine Folding. J. Chem. Phys. 1995, 103, 773-787.

(11) Miyazawa, S.; Jernigan, R. L. An Empirical Energy Potential with a Reference State for Protein Fold and Sequence Recognition. Proteins: Struct., Funct., Bioinf. 1999, 36, 357-369. 